\title{
Role of allostatic load in socio-demographic patterns of pain prevalence in the US population
}

\author{
Gary D. Slade ${ }^{1,2}$, Anne E. Sanders ${ }^{1}$, and Kunthel By ${ }^{3}$ \\ ${ }^{1}$ Center for Neurosensory Disorders, University of North Carolina at Chapel Hill, Chapel Hill, NC \\ ${ }^{2}$ Department of Epidemiology, University of North Carolina at Chapel Hill, Chapel Hill, NC \\ ${ }^{3}$ Department of Biostatistics, University of North Carolina at Chapel Hill, Chapel Hill, NC
}

\section{Abstract}

Persistent stressors associated with sociodemographic disadvantage exert a physiologic toll, labeled "allostatis load", that contributes to disparities in some health conditions. We investigated the contribution of allostatic load to pain prevalence in US adults. Interviews with 14,184 adults in the 1999-2004 National Health and Nutrition Examination Survey asked about severe headache, pain that lasted $>24$ hours, and widespread pain. Ten biomarkers of allostatic load were quantified from blood (glycated hemoglobin), serum (C-reactive protein, homocysteine, cholesterol, triglycerides), urine (creatinine, albumin) and physical measurements (body mass index, systolic and diastolic blood pressure). Log-binomial regression models estimated prevalence ratios (PR) and $95 \%$ confidence intervals (95\%CIs). Prevalence ranged from $3.4 \%$ for widespread pain to $26.9 \%$ for pain $>24$ hours. After adjustment for demographic characteristics, low income was associated with greater prevalence of pain $>24$ hours $(\mathrm{PR}=1.65,95 \% \mathrm{CI}=1.49,1.83)$, severe headache $(\mathrm{PR}=2.05,95 \% \mathrm{CI}=1.68,2.50)$ and widespread pain $(\mathrm{PR}=3.67,95 \% \mathrm{CI}=2.56,5.27)$. Racial/ethnic minorities had lower prevalence of all three pain conditions than non-Hispanic Whites. While greater allostatic load was associated with elevated prevalence of pain, allostatic load did not meaningfully attenuate prevalence ratios associated with income or race/ethnicity. We conclude that greater pain prevalence among low income groups is not explained by greater allostatic load.

\section{Keywords}

pain; epidemiology; allostatic load; US population; socioeconomic factors

\section{Introduction}

Like many other persistent health conditions, chronic pain occurs in response to a complex interplay of environmental and genetic influences that alter biological and psychological

\footnotetext{
(C) 2012 The American Pain Society. Published by Elsevier Inc. All rights reserved.

Author for correspondence: Gary D. Slade, BDSc, DDPH, PhD, Old Dental Building, Room 2100, UNC School of Dentistry, CB \#7450, Chapel Hill, NC 27599-7450, USA. Phone: [Int +1] 919-843-0419. FAX: 919-843-1170. gary_slade@ dentistry.unc.edu.

Publisher's Disclaimer: This is a PDF file of an unedited manuscript that has been accepted for publication. As a service to our customers we are providing this early version of the manuscript. The manuscript will undergo copyediting, typesetting, and review of the resulting proof before it is published in its final citable form. Please note that during the production process errors may be discovered which could affect the content, and all legal disclaimers that apply to the journal pertain.

Disclosures

The authors declare no financial or other relationships that might lead to a conflict of interest in their conduct of this research. The authors declare no conflicts of interest in their conduct of this research.
} 
regulatory systems. For example, one conceptual etiologic model of idioapthic pain conditions depicts two primary pathways of vulnerability: pain amplification and psychological distress. ${ }^{14}$ In this study, we investigated an additional hypothesized pathway of allostatic load.

The concept of allostasis, a complex form of physiological regulation, was developed to explain pathologic change in regulatory systems that occurs in response to repeated arousal. ${ }^{54}$ Unlike homeostasis which limits change by holding constant parameters essential for life — such as body temperature, $\mathrm{pH}$, and oxygen tension-allostasis evokes change essential to adaptation. ${ }^{54}$ Multiple regulatory systems are involved including the hypothalamic-pituitary-adrenocortical (HPA) axis, sympathetic and parasympathetic nervous system and immune system. In the short-term, stress mediators from these systems promote adaptation and the response protects health. However sustained or repeated deviation from regular physiological parameters produces a biological burden referred, to as allostatic load, ${ }^{35}$ that is maladaptive across a range of biological regulatory systems. Accordingly, studies have used numerous biomarkers to index the degree of allostatic load. ${ }^{10,17,47,48}$

Investigations of stressors contributing to allostatic load implicate low socioeconomic status (SES) and experiences of racial/ethnic-disadvantage. Low SES is associated with greater exposure to health risk behaviors as well as financial strain, depression, anxiety, discrimination and boredom. ${ }^{22,30,45,50}$ In the United States, non-Hispanic Blacks experience disproportionately low levels of SES. Many of them also experience effects of racism, segregation and discrimination that represent additional, long term hazards to health. ${ }^{3}$ The pervasive nature of these experiences and repeated efforts to adapt to them are thought to build allostatic load. For example, groups with low SES have elevated levels of stress hormones such as cortisol, epinephrine and norepinephrine. ${ }^{9}$ Many adults in the US have low income and/or experience racial/ethnic disadvantage. Such groups may be more susceptible than other groups to highly prevalent conditions, such as chronic pain, because of the continuous nature of stress that they confront, and the allostatic load that ensues. .

Furthermore, there is good evidence from large, population-based samples that prevalence of pain disorders is greater in socioeconomically disadvantaged groups. A national study of neck and lower back pain conducted in Spain, for instance, found inverse monotonic gradients in prevalence of both conditions across levels of education and income. ${ }^{16}$ Likewise low household income and unemployment were significantly associated with chronic, recurrent or long-lasting pain in a representative sample of US adults. ${ }^{27}$ Similar socioeconomic associations with pain are reported in the Canadian National Population Health Survey. ${ }^{42}$ and in Singapore. ${ }^{58}$

Cross-sectional evidence shows that high allostatic load is associated with cardiovascular disease, ${ }^{32,43}$ periodontal disease, ${ }^{43}$ and chronic fatigue syndrome ${ }^{18}$. In longitudinal studies, high allostatic load is predictive of all cause mortality ${ }^{3}$ and declines in cognitive and physical functioning. ${ }^{46}$ Whether allostatic load contributes to pain is uncertain, but nonetheless plausible. For instance, HPA axis activity plays a role in stress-related pain disorders including chronic headache, chronic pelvic pain, fibromyalgia, chronic fatigue syndrome and irritable bowel syndrome. ${ }^{11,13,21,56}$

In this study, we evaluate SES and racial/ethnic group variation in prevalence of commonlyreported pain symptoms and assess associations between biomarkers of allostatic load and prevalence of pain in the US adult population. We hypothesized that an index of allostatic load should account for some or all of the SES and racial/ethnic group gradient in prevalence of pain symptoms. 


\section{Materials and Methods}

\section{Study design and population}

The National Health and Nutrition Examination Survey (NHANES) is a cross-sectional study designed to provide information on the distribution of various health and nutritional outcomes as well as potential risk factors. The target population consists of the noninstitutionalized population aged 2 months or older living in the 50 US states, District of Columbia, and Puerto Rico. NHANES uses a complex, multistage, probability design to obtain a representative sample of the civilian, non-institutionalized US population. The primary sampling units (PSUs) are predominantly counties. Segments of these PSUs are sampled, and from within these segments, a random sample of households is drawn. Each year NHANES examines about 5,000 persons located in counties across the country. Data are released in two-year cycles. This paper analyzes data from 1999 - 2004 NHANES cycles $^{5}$ in which 14,184 adults 18 years or older completed questions about pain and provided blood that was used to measure biomarkers of allostatic load.

\section{Classification of three pain conditions}

Study participants were classified according to their response to questions about three pain conditions included in the NHANES Miscellaneous Pain Questionnaire ${ }^{8}$. Participants were classified as having severe headache if they responded "yes" to the question, "During the past 3 months, did you have severe headaches or migraines?" Those responding affirmatively to the question "During the past month, have you had a problem with pain that lasted more than 24 hours?" were classified as having experience pain for more than 24 hours (pain $>24$ hours). Among these subjects, they were classified as having widespread bodily pain (WBP) if pain was present in the upper right, upper left, lower right, lower left, and axial regions of the body ${ }^{20}$ (p. 805, 1st paragraph). Participants indicated regions of the body that were affected by pain by pointing to a manikin drawn on a card. Interviewers encoded responses using 32 pre-coded anatomical descriptions. Respondents reporting fewer affected sites and those who did not have pain $>24$ hours were classified as non-cases of WBP.

\section{Index of allostatic load}

The NHANES study measured 10 biomarkers of allostatic load because these are used widely as an index of allosatic load in epidemiologic studies. ${ }^{10,17,47,48}$ The biomarkers were quantified from blood (glycated hemoglobin), serum (C-reactive protein, homocysteine, cholesterol, triglycerides), urine (creatinine, albumin) and physical measurements (body mass index, systolic and diastolic blood pressure). SBP, DBP, and BMI were obtained from physical examinations ${ }^{6}$ and the others were obtained from laboratory examinations. ${ }^{7}$ Consistent with the method used by Geronimus et al. ${ }^{17}$ to create an index of allostatic load, subjects were classified into quartiles for each of the ten biomarkers. An individual was classified at higher risk for allostatic load if the level of the biomarker was in the lowest quartile for albumin and urinary creatinine clearance and in the highest quartile for the other eight biomarkers, namely: serum albumin $<4.08 \mathrm{~g} / \mathrm{dL}$; BMI $\geq 31.16$; serum Creactive protein $\geq 0.45 \mathrm{mg} / \mathrm{dL}$; urinary creatinine clearance $<65.05 \mathrm{mg} / \mathrm{dL}$; diastolic blood pressure $\geq 78.73 \mathrm{~mm} \mathrm{Hg}$; systolic blood pressure $\geq 131.15 \mathrm{~mm} \mathrm{Hg}$; serum glycated hemoglobin $\geq 5.5 \%$; serum homocysteine $\geq 9.79 \mu \mathrm{mol} / \mathrm{L}$; total serum cholesterol $\geq 225.96$ $\mathrm{mg} / \mathrm{dL}$; serum triglycerides $>166.59 \mathrm{mg} / \mathrm{dL}$. The allostatic load index for each individual is defined as the total number of biomarkers at the highest risk quartile. Individuals with a missing value for any component of the allostatic load index were excluded from analysis.

Covariates-Analyses considered the following socio-demographic covariates and risk factors: age, gender, race/ethnicity, income (expressed as the ratio of income to the poverty 
level, i.e. PIR), and smoking status. Smoking was included because of its recognized relationship with multiple chronic pain conditions $36,44,49$. Age was defined as a categorical variable with seven levels: $18-24,25-34,35-44,45-54,55-64,65-74$, and $\geq 75$ years. Four categories defined race/ethnicity: Non-Hispanic White (NHW), Non-Hispanic Black (NHB), Hispanic, and Other. With respect to smoking status, individuals were classified as either current, former, or never.

SES was operationalized using the ratio of family income to poverty. This widely-cited derived continuous variable on the NHANES dataset divides family income by the poverty threshold, taking into account the family size. As such it represents equivalized income which is more informative about available resources than income alone. A poverty-income ratio below 1.0 indicates that the family is below the poverty threshold. Hereafter we refer to poverty-income ratio simply as "income". For this analysis, income was defined as a categorical variable with the following levels: low income $(<1.0)$, medium (from 1.0 up to but not including the estimated third quartile, 4.79), and high (at or above the estimated third quartile).

\section{Statistical analysis}

Statistical approaches appropriate for complex multi-stage sample surveys were used in all data analyses, including use of sampling design variables (stratum and primary sampling units) and sampling weights for examined subjects. Variances of estimated quantities are computed using Taylor series approximations ${ }^{231}$. Prevalence proportions and prevalence ratios were estimated using log-binomial regression models 51,19 and their standard errors were estimated using results established by Natarajan et al ${ }^{37}$.

Unadjusted estimates of pain prevalence for each of the three pain outcomes were computed for groups classified according to the following risk factors and socio-demographic variables: smoking status, gender, age, race/ethnicity, and PIR. Significance was assessed using Rao-Scott ${ }^{41}$ chi-squares.

Demographically-adjusted prevalence estimates across levels of the allostatic load index were estimated by fitting a log-binomial regression model treating the allostatic load index as a categorical variable with six levels $(0,1,2.3,4,25)$ and adjusting for the following demographic factors: age, gender, and race/ethnicity. For each of the 10 biomarkers used to define the allostatic load index, we also fitted a demographically-adjusted log-binomial regression model treating the biomarker as a categorical variable with three levels: less than the first quartile, from the first quartile up to but not including the third quartile, and at or above the third quartile. Significance was assessed using Wald chi-squares.

For each of the three pain conditions, prevalence ratios comparing low and medium to high PIR were estimated by fitting a series of three log-binomial regression models adjusting for age, gender, and race/ethnicity with a view towards assessing attenuation in these ratios, if any. Each successive model included an additional risk factor (smoking status and allostatic load index as a 6-level categorical variable).. Wald confidence intervals were computed for prevalence ratios in the log scale and then exponentiated to obtain confidence intervals for prevalence ratios.

\section{Results}

\section{Socio-demographic variation in prevalence of pain}

Widespread bodily pain was experienced by an estimated $3.4 \%$ of US adults, while more than one fifth reported severe headache $(21.6 \%)$ or pain $>24$ hours $(26.9 \%)$ (Table 1). For all three types of reported pain, prevalence was greater for females than for males, and it was 
higher for age groups within the range 25-64 years compared to the oldest- or youngest-agegroups. In these unadjusted analyses, pain $>24$ hours occurred more frequently in nonHispanic Whites than other race/ethnic groups, whereas severe headache had greatest prevalence in Hispanics. Prevalence of the three pain conditions was inversely associated with income, and current smokers had greater prevalence of these pain conditions than either former-smokers or people who had never smoked.

\section{Associations between allostatic mediators and pain}

In analyses that adjusted for age, gender and race/ethnicity, severe headache and pain $>24$ hours each were associated with most individual biomarkers of allostatic load (Table 2). Greater prevalence generally was observed in people with markers of high allostatic load. Specifically, prevalence of both severe headache and pain $>24$ hours was positively associated with measures of body mass index, serum C-reactive protein, glycated hemoglobin and serum triglycerides. Prevalence of both severe headache and pain $>24$ hours was negatively associated with serum albumin concentration, signifying higher allostatic load. However greater prevalence of severe headache was associated with higher values of urinary creatinine clearance, signifying low allostatic load. Demographically-adjusted prevalence of WBP generally displayed similar patterns of association with the same markers of allostatic load, although net differences in prevalence among quartiles of each marker were small, and only three reached statistical significance (body mass index, serum C-reactive protein and serum triglycerides). Prevalence of all three pain conditions was positively associated with higher scores of the allostasis index (Table 2), although the relationship was not monotonic. However for each pain condition, people who scored at least 5 on the index had greater prevalence than people with lower scores.

\section{Multivariable analysis of associations between allostatic mediators and pain}

Without adjustment for covariates, the mean allostatic load index was inversely associated with income, and it was greater in non-Hispanic Blacks than in non-Hispanic Whites (results not tabulated). For example, the low income group had a higher allostatic load (mean index $=2.64,95 \% \mathrm{CI}=2.52,2.76)$ than the high income group (mean index $=2.34,95 \% \mathrm{CI}=2.25$, 2.45). Relative to non-Hispanic Whites (mean index $=2.55,95 \% \mathrm{CI}=2.46,2.63$ ), allostatic load was greater in non-Hispanic Blacks (mean index $=2.75,95 \% \mathrm{CI}=2.66,2.86$ ), although it was lower in Hispanics (mean index $=2.33,95 \% \mathrm{CI}=2.21,2.46$ ).

Demographically-adjusted pain prevalence ratios for the lowest income category relative to the highest income category ranged from 1.65 for pain $>24$ hours to 3.67 for WBP (Table 3, Model 1). For each pain condition, the middle-income category had a lower prevalence ratio, and corresponding 95\% CIs excluded the null value of 1.0. In contrast, prevalence ratios for racial/ethnic groups showed no significant variation in prevalence of severe headache, while for the other two forms of pain, racial/ethnic group minorities each had lower prevalence relative to the reference group of non-Hispanic Whites.

There were only small changes in prevalence ratios for categories of income after additionally adjusting for smoking. Associations of income with each pain condition remained statistically significant, and relative to non-Hispanic Whites, racial/ethnic minorities continued to have significantly lower prevalence of pain $>24$ hours and widespread bodily pain (Table 3, Model 2). Likewise, prevalence ratios were only modestly attenuated after additional adjustment for the allostatic load index (Model 3). The largest amount of attenuation was seen for WBP, where the prevalence ratio for the lowest- relative to the highest-income category was $3.67(95 \% \mathrm{CI}=2.56,5.27)$ in the demographicallyadjusted model 1 and $2.97(2.16,4.10)$ in the fully-adjusted model 3 . Because the $95 \%$ CIs in each model overlapped to a large degree, we interpret this as only modest attenuation of the 
prevalence ratio. Likewise, there was little change in the magnitude of protective prevalence ratios for racial/ethnic minorities relative to non-Hispanic Whites for pain $>24$ hours and for widespread bodily pain. In Model 3, the fully-adjusted association of the allostatic load index was only modest for severe headache (prevalence ratio $=1.23(1.02,1.47)$ for a score of $\geq 5$ relative to a score of 0 ) and for pain $>24$ hours (prevalence ratio $=1.45(1.22,1.73$ ). However, for widespread pain, prevalence ratios were larger, and statistically significant for each level of the allostatic load index above the reference level of zero.

\section{Discussion}

In this cross-sectional study of US adults, prevalence of all three commonly-occurring pain symptoms (severe headache, pain $\geq 24$ hours, widespread bodily pain) was positively associated with greater allostatic load. Despite a pattern of greater pain prevalence and greater allostatic load in lower income groups, allostatic load did not account for the relationship between income and pain prevalence. Furthermore, although prevalence of pain $>24$ hours and of widespread bodily pain was lower in racial/ethnic minorities than in nonHispanic Whites, this pattern was not altered after adjusting for allostatic load. While these findings support the notion that pain is one consequence of the biologic "wear and tear" induced by low SES, these results do not support the hypothesis that allostatic load accounts for SES variation in self-reported pain.

Because of its cross-sectional design, this study is unable to compute people's risk of developing pain, and nor is it possible to establish a temporal sequence between elevation in biomarkers and onset of pain. Yet the relationship between allostatic load and pain symptoms is consistent with other evidence that psychological and behavioral stressors contribute to chronic pain, ${ }^{28}$ an effect that is mediated primarily through the HPA axis. ${ }^{34}$ Dysregulation of the HPA axis, a hallmark of allostatic load, ${ }^{33}$ can be quantified using measures of corticotrophin-releasing factor, adrenocorticotropin and glucococoricoids. Indeed, McEwen and Seeman conceived of HPA mediators as "primary" mediators of allostatic load because they signify the first change in a sequence of events. Specifically, primary mediators measure cellular events and activity of messenger systems. ${ }^{35}$ In the same conceptual model, ${ }^{35}$ those first changes lead to secondary changes in metabolic processes and preclinical signs of pathology such as glycosylation of hemoglobin and alterations to serum lipids. HPA axis mediators were not measured in the NHANES study. Instead, we used an index comprised of secondary allostatic mediators because previous studies found them to be elevated in non-Hispanic Blacks and in low income groups, ${ }^{17}$ a result that we confirmed using these NHANES 1999-2004 data.

In this study, C-reactive protein was the allostatic load biomarker most strongly associated with pain symptoms. Although still within "normal" limits, these elevated C-reactive protein levels signify a general pro-inflammatory state. High-normal serum concentrations of Creactive protein are associated with many other diseases that have an inflammatory component, including cardiovascular disease. ${ }^{29}$ The association observed here is consistent with evidence regarding the role of pro-inflammatory cytokines in some types of chronic pain, most notably fibromyalgia ${ }^{591}$ In this study, elevated CRP was associated with greater prevalence of all three pain conditions. In the multivariable models, the association between the overall allostatic load index and pain was greatest for widespread bodily pain. The observed prevalence was based on a stringent case-definition for widespread pain that yielded overall prevalence of $3.4 \%$ which is lower than rates of $5 \%^{24}$ to $11 \%{ }^{12}$ reported using the Manchester definition. The difference is probably due to the NHANES-criterion that required widespread pain of more than 24 hours duration in the preceding month. Greater stringency in case-classification can alter strengths of association with putative risk 
factors. For example, Hunt et $\mathrm{al}^{24}$ found stronger associations between psychosocial factors and pain using the Manchester definition compared to a less stringent case-definition.

We therefore propose two explanations for the observed associations between this allostatic load index and pain symptoms. For symptoms of widespread bodily pain, where inflammation is a likely contributing risk factor, we believe the strong association with the allostatic load index occurs because one of the index's components is a direct measure of inflammatory burden (C-reactive protein); the remaining components are secondary allostatic mediators, thereby providing an additional, indirect marker of HPA axis dysregulation. In contrast, for headache and pain $>24$ hours, inflammation probably is less critical, such that the association with the index of allostatic load occurs only indirectly, to the extent that secondary indicators of allostatic load signify alterations in the HPA axis. However, these interpretations are speculative. The NHANES laboratory protocol did not include direct biomarkers of HPA axis function that have been used in other studies. ${ }^{48}$ Hence, this study's findings of generally weak associations between these 10 secondary allostatic mediators and pain do not negate the possibility that primary allostatic mediators might show strong associations with pain. Indeed, we recommend that future research into this question focus on primary allostatic mediators.

The observed lack of attenuation of the SES gradient by the allostatic load index suggests that low SES contributes to pain symptoms primarily through pathways that do not involve allostatic load. This is in contrast to the notion that the stress of low SES imposes physiological wear-and-tear that contributes to poor health. While that notion has been supported by experimental studies of cardiovascular disease and its precursors, ${ }^{5253}$, observational epidemiological studies have not necessarily confirmed that allostatic load is responsible for all or some of the SES gradient in cardiovascular disease and other disorders. ${ }^{43}$

The evidence is also ambiguous with respect to the role of allostatic load in explaining racial/ethnic differences in disease in the United States. ${ }^{3}$ At first appearance, the lower prevalence of two pain conditions observed here in racial/ethnic minorities casts further doubt on the salience of allostatic load as a mediator of racial-group variation in pain. The finding of similar or lower pain prevalence in racial/ethnic minorities compared to nonHispanic whites is consistent with nationally-representative studies that have investigated migraine ${ }^{55}$, non-specific headache, neck pain and back pain ${ }^{39}$ and temporomandibular disorder and neck pain. ${ }^{26,39}$ Yet, racial/ethnic minorities typically report greater sensitivity to experimental pain, ${ }^{15}$ and there is evidence that racial/ethnic minorities are disadvantaged in receiving treatment for pain. 40

Given that allostatic load is elevated in US racial/ethnic minorities relative to non-Hispanic whites,,${ }^{17}$ it is implausible that accounting for allostatic load in multivariable modeling might reverse the observed pattern of lower pain prevalence in minorities. However, before dismissing the relevance of allostatic load in understanding racial/ethnic variation in pain prevalence, it should be emphasized that it is the experience and perceptions of disadvantage that are thought to add to allostatic load, rather than racial/ethnic identity itself. It is plausible that measures of discrimination or other socially-patterned forms of disadvantage might be associated with greater prevalence of pain. If that was the case, allostatic load might constitute a mediator of the relationship. However, this idea cannot be tested using these data because the NHANES survey did not measure racial/ethnic discrimination.

Smoking rates are negatively related to income in the US 4 and in this study smoking was positively associated with prevalence of pain. We therefore investigated whether smoking accounted for income disparities in self-reported pain. While prevalence of all three pain 
conditions was highest among people with low income and among current smokers, statistical adjustment for smoking did not attenuate income-associated prevalence ratio for severe headache or for pain $>24$ hours; and there was only modest reduction of the prevalence ratio for widespread bodily pain. Likewise, adjustment for smoking did not alter racial/ethnic differences in self-reported pain. Consequently we conclude that while smoking may be a risk indicator for pain, smoking does not account for differences in variations in pain prevalence associated with income and with racial/ethnic identity.

An important consideration when interpreting these findings is that the pain symptoms were reported only during standardized interviews - there were no clinical assessments to determine specific pain diagnoses. Hence, these reports of headache do not conform with specific diagnostic categories of headache ${ }^{23}$, and reported pain in multiple body sites is not equivalent to fibromyalgia. ${ }^{57}$ Nonetheless, global assessments of pain symptoms have public health relevance by serving as indicators of everyday-pain experiences within the population. Indeed, simple, interview-based measures for assessing widespread bodily pain have been advocated as a pragmatic way of investigating population-level distribution and determinants of chronic pain. ${ }^{38}$ A notable benefit of population-based studies, such as NHANES, is that they do not rely on attendance or referral to specialists, who typically are needed to render diagnoses of specific chronic pain conditions. Indeed, the US Institute of Medicine ${ }^{25}$ noted that national surveillance systems such as NHANES are an essential tool in monitoring the public health burden created by chronic pain.

In summary, these findings show that greater prevalence of commonly-occurring pain symptoms is associated with increasing levels of allostatic load, as assessed using secondary mediators of allostatic load. Yet, greater allostatic load in low SES groups did not account for their greater prevalence of pain. And conversely, despite greater allostatic load among racial/ethnic minorities, they had lower prevalence of pain than non-Hispanic Whites. These findings are consistent with some studies of other chronic diseases by casting doubt on the salience of secondary mediators of allostatic load in explaining SES variation in commonlyoccurring pain symptoms. While it is possible that primary allostatic mediators, such as cortisol and catecholamines, might provide an index of allostatic load that is more salient for these forms of pain, it is possible that other biological, behavioral, or psychological processes account for SES variation in pain prevalence in the US population.

\section{Acknowledgments}

This study was supported by NIH/National Institute of Neurological Disorders and Stroke. 2 P01 NS045685.

\section{References}

1. Bazzichi L, Rossi A, Massimetti G, Giannaccini G, Giuliano T, De Feo F, Ciapparelli A, Dell'Osso L, Bombardieri S. Cytokine patterns in fibromyalgia and their correlation with clinical manifestations. Clinical and experimental rheumatology. 2007; 25:225-30. [PubMed: 17543146]

2. Binder DA. On the Variances of Asymptotically Normal Estimators from Complex Surveys. Int Stat Rev. 1983; 51:279-92.

3. Borrell LN, Dallo FJ, Nguyen N. Racial/ethnic disparities in all-cause mortality in U.S. adults: the effect of allostatic load. Public Health Rep. 2010; 125:810-6. [PubMed: 21121226]

4. Centers for Disease Control and Prevention (CDC). Any tobacco use in 13 States --- behavioral risk factor surveillance system, 2008. MMWR Morb Mortal Wkly Rep. 2010; 59:946-50. [PubMed: 20689499]

5. Centers for Disease Control and Prevention (CDC). [August 11, 2011] National Center for Health Statistics (NCHS): National Health and Nutrition Examination Survey Data. http://www.cdc.gov/ nchs/nhanes.htm. 
6. Centers for Disease Control and Prevention (CDC). [August 11, 2011] National Center for Health Statistics (NCHS): National Health and Nutrition Examination Survey Examination Protocol. http:// www.cdc.gov/nchs/nhanes.htm.

7. Centers for Disease Control and Prevention (CDC). [August 11, 2011] National Center for Health Statistics (NCHS): National Health and Nutrition Examination Survey Laboratory Protocol. http:// www.cdc.gov/nchs/nhanes.htm

8. Centers for Disease Control and Prevention (CDC). [August 11, 2011] National Center for Health Statistics (NCHS): National Health and Nutrition Examination Survey Questionnaire. http:// www.cdc.gov/nchs/nhanes.htm

9. Cohen S, Doyle WJ, Baum A. Socioeconomic status is associated with stress hormones. Psychosom Med. 2006; 68:414-20. [PubMed: 16738073]

10. Crimmins EM, Johnston M, Hayward M, Seeman T. Age differences in allostatic load: an index of physiological dysregulation. Exp Gerontol. 2003; 38:731-4. [PubMed: 12855278]

11. Crofford LJ, Young EA, Engleberg NC, Korszun A, Brucksch CB, McClure LA, Brown MB, Demitrack MA. Basal circadian and pulsatile ACTH and cortisol secretion in patients with fibromyalgia and/or chronic fatigue syndrome. Brain Behav Immun. 2004; 18:314-25. [PubMed: 15157948]

12. Croft P, Rigby AS, Boswell R, Schollum J, Silman A. The prevalence of chronic widespread pain in the general population. J Rheumatol. 1993; 20:710-3. [PubMed: 8496870]

13. Demitrack MA, Crofford LJ. Evidence for and pathophysiologic implications of hypothalamicpituitary-adrenal axis dysregulation in fibromyalgia and chronic fatigue syndrome. Ann N Y Acad Sci. 1998; 840:684-97. [PubMed: 9629295]

14. Diatchenko L, Nackley AG, Slade GD, Fillingim RB, Maixner W. Idiopathic pain disorders-pathways of vulnerability. Pain. 2006; 123:226-30. [PubMed: 16777329]

15. Edwards CL, Fillingim RB, Keefe F. Race, ethnicity and pain. Pain. 2001; 94:133-7. [PubMed: 11690726]

16. Fernandez-de-las-Penas C, Hernandez-Barrera V, Alonso-Blanco C, Palacios-Cena D, CarrascoGarrido P, Jimenez-Sanchez S, Jimenez-Garcia R. Prevalence of neck and low back pain in community-dwelling adults in Spain: a population-based national study. Spine (Phila Pa 1976). 2011; 36:E213-9. [PubMed: 21079541]

17. Geronimus AT, Hicken M, Keene D, Bound J. "Weathering" and age patterns of allostatic load scores among blacks and whites in the United States. Am J Public Health. 2006; 96:826-33. [PubMed: 16380565]

18. Goertzel BN, Pennachin C, de Souza Coelho L, Maloney EM, Jones JF, Gurbaxani B. Allostatic load is associated with symptoms in chronic fatigue syndrome patients. Pharmacogenomics. 2006; 7:485-94. [PubMed: 16610958]

19. Greenland S. Model-based estimation of relative risks and other epidemiologic measures in studies of common outcomes and in case-control studies. Am J Epidemiol. 2004; 160:301-5. [PubMed: $15286014]$

20. Hardt J, Jacobsen C, Goldberg J, Nickel R, Buchwald D. Prevalence of chronic pain in a representative sample in the United States. Pain Med. 2008; 9:803-12. [PubMed: 18346058]

21. Harris RE, Sundgren PC, Craig AD, Kirshenbaum E, Sen A, Napadow V, Clauw DJ. Elevated insular glutamate in fibromyalgia is associated with experimental pain. Arthritis Rheum. 2009; 60:3146-52. [PubMed: 19790053]

22. Hawkley LC, Lavelle LA, Berntson GG, Cacioppo JT. Mediators of the relationship between socioeconomic status and allostatic load in the Chicago Health, Aging, and Social Relations Study (CHASRS). Psychophysiology. 2011

23. Headache Classification Subcommittee of the International Headache Society: The International Classification of Headache Disorders: 2nd edition. Cephalalgia. 2004; 24(Suppl 1):9-160. [PubMed: 14979299]

24. Hunt IM, Silman AJ, Benjamin S, McBeth J, Macfarlane GJ. The prevalence and associated features of chronic widespread pain in the community using the 'Manchester' definition of chronic widespread pain. Rheumatology (Oxford). 1999; 38:275-9. [PubMed: 10325667] 
25. IOM (Institute of Medicine): Relieving Pain in America: A Blueprint for Transforming Prevention, Care, Education, and Research. The National Academies Press; Washington, DC: 2011.

26. Isong U, Gansky S, Plesh O. Temporomandibular joint and muscle disorder-type pain in U.S. adults: the National Health Interview Survey. J Orofac Pain. 2008; 22:317-22. [PubMed: 19090404]

27. Johannes CB, Le TK, Zhou X, Johnston JA, Dworkin RH. The prevalence of chronic pain in United States adults: results of an Internet-based survey. J Pain. 2010; 11:1230-9. [PubMed: 20797916]

28. Jones, AKP.; McBeth, J.; Power, A. The biological response to stress and chronic pain.. In: Croft, P.; Blyth, FM.; Windt, Dvd, editors. Chronic pain epidemiology : from aetiology to public health. Oxford University Press; Oxford ; New York: 2010. p. 101-18.

29. Kaptoge S, Di Angelantonio E, Lowe G, Pepys MB, Thompson SG, Collins R, Danesh J. Creactive protein concentration and risk of coronary heart disease, stroke, and mortality: an individual participant meta-analysis. Lancet. 2010; 375:132-40. [PubMed: 20031199]

30. Kubzansky LD, Kawachi I, Sparrow D. Socioeconomic status, hostility, and risk factor clustering in the Normative Aging Study: any help from the concept of allostatic load? Ann Behav Med. 1999; 21:330-8. [PubMed: 10721441]

31. Lohr, SL. Sampling : design and analysis. Duxbury Press; Pacific Grove, CA: 1999.

32. Mattei J, Demissie S, Falcon LM, Ordovas JM, Tucker K. Allostatic load is associated with chronic conditions in the Boston Puerto Rican Health Study. Soc Sci Med. 2010; 70:1988-96. [PubMed: 20381934]

33. McEwen BS. Physiology and neurobiology of stress and adaptation: central role of the brain. Physiological reviews. 2007; 87:873-904. [PubMed: 17615391]

34. McEwen BS, Kalia M. The role of corticosteroids and stress in chronic pain conditions. Metabolism: clinical and experimental. 2010; 59(Suppl 1):S9-15. [PubMed: 20837196]

35. McEwen BS, Seeman T. Protective and damaging effects of mediators of stress. Elaborating and testing the concepts of allostasis and allostatic load. Ann N Y Acad Sci. 1999; 896:30-47. [PubMed: 10681886]

36. Mitchell MD, Mannino DM, Steinke DT, Kryscio RJ, Bush HM, Crofford LJ. Association of smoking and chronic pain syndromes in kentucky women. J Pain. 2011; 12:892-9. [PubMed: 21816352]

37. Natarajan S, Lipsitz SR, Fitzmaurice G, Moore CG, Gonin R. Variance estimation in complex survey sampling for generalized linear models. Journal of the Royal Statistical Society Series CApplied Statistics. 2008; 57:75-87.

38. Natvig, B.; Ihlebaek, C.; Kamaleri, Y.; Bruusgaard, D. Number of pain sties - a simple measure of population risk?. In: Croft, P.; Blyth, FM.; Windt, Dvd, editors. Chronic pain epidemiology : from aetiology to public health. Oxford University Press; Oxford ; New York: 2010. p. 71-9.

39. Plesh O, Adams SH, Gansky SA. Racial/Ethnic and gender prevalences in reported common pains in a national sample. Journal of orofacial pain. 2011; 25:25-31. [PubMed: 21359234]

40. Portenoy RK, Ugarte C, Fuller I, Haas G. Population-based survey of pain in the United States: differences among white, African American, and Hispanic subjects. The journal of pain : official journal of the American Pain Society. 2004; 5:317-28. [PubMed: 15336636]

41. Rao JNK, Scott AJ. The Analysis of Categorical-Data from Complex Sample-Surveys - ChiSquared Tests for Goodness of Fit and Independence in 2-Way Tables. Journal of the American Statistical Association. 1981; 76:221-30.

42. Rashiq S, Dick BD. Factors associated with chronic noncancer pain in the Canadian population. Pain Res Manag. 2009; 14:454-60. [PubMed: 20011716]

43. Sabbah W, Watt RG, Sheiham A, Tsakos G. Effects of allostatic load on the social gradient in ischaemic heart disease and periodontal disease: evidence from the Third National Health and Nutrition Examination Survey. J Epidemiol Community Health. 2008; 62:415-20. [PubMed: 18413454]

44. Sanders AE, Slade GD, Maixner W, Nackley AG, Diatchenko L, By K, Miller VE. Excess Risk of Temporomandibular Disorder Associated with Cigarette Smoking in Young Adults. J Pain. 
45. Seeman TE, Crimmins E, Huang MH, Singer B, Bucur A, Gruenewald T, Berkman LF, Reuben DB. Cumulative biological risk and socio-economic differences in mortality: MacArthur studies of successful aging. Soc Sci Med. 2004; 58:1985-97. [PubMed: 15020014]

46. Seeman TE, McEwen BS, Rowe JW, Singer BH. Allostatic load as a marker of cumulative biological risk: MacArthur studies of successful aging. Proc Natl Acad Sci U S A. 2001; 98:47705. [PubMed: 11287659]

47. Seeman TE, Singer B, Wilkinson CW, McEwen B. Gender differences in age-related changes in HPA axis reactivity. Psychoneuroendocrinology. 2001; 26:225-40. [PubMed: 11166486]

48. Seeman TE, Singer BH, Rowe JW, Horwitz RI, McEwen BS. Price of adaptation--allostatic load and its health consequences. MacArthur studies of successful aging. Arch Intern Med. 1997; 157:2259-68. [PubMed: 9343003]

49. Shiri R, Karppinen J, Leino-Arjas P, Solovieva S, Viikari-Juntura E. The association between smoking and low back pain: a meta-analysis. Am J Med. 2010; 123:87, e7-35. [PubMed: 20102998]

50. Singer B, Ryff CD. Hierarchies of life histories and associated health risks. Ann N Y Acad Sci. 1999; 896:96-115. [PubMed: 10681891]

51. Spiegelman D, Hertzmark E. Easy SAS calculations for risk or prevalence ratios and differences. American Journal of Epidemiology. 2005; 162:199-200. [PubMed: 15987728]

52. Steptoe A, Feldman PJ, Kunz S, Owen N, Willemsen G, Marmot M. Stress responsivity and socioeconomic status: a mechanism for increased cardiovascular disease risk? European heart journal. 2002; 23:1757-63. [PubMed: 12419295]

53. Steptoe A, Marmot M. The role of psychobiological pathways in socio-economic inequalities in cardiovascular disease risk. European heart journal. 2002; 23:13-25. [PubMed: 11741358]

54. Sterling, P.; Eyer, J. Allostasis: A New Paradigm to Explain Arousal Pathology.. In: Fisher, S.; Reason, J., editors. Handbook of Life Stress, Cognition and Health. John Wiley \& Sons Ltd; New York: 1988. p. 629-49.

55. Stewart WF, Lipton RB, Celentano DD, Reed ML. Prevalence of migraine headache in the United States. Relation to age, income, race, and other sociodemographic factors. JAMA. 1992; 267:64-9. [PubMed: 1727198]

56. Tanriverdi F, Karaca Z, Unluhizarci K, Kelestimur F. The hypothalamo-pituitary-adrenal axis in chronic fatigue syndrome and fibromyalgia syndrome. Stress. 2007; 10:13-25. [PubMed: 17454963]

57. Wolfe F, Clauw DJ, Fitzcharles MA, Goldenberg DL, Katz RS, Mease P, Russell AS, Russell IJ, Winfield JB, Yunus MB. The American College of Rheumatology preliminary diagnostic criteria for fibromyalgia and measurement of symptom severity. Arthritis care \& research. 2010; 62:60010. [PubMed: 20461783]

58. Yeo SN, Tay KH. Pain prevalence in Singapore. Ann Acad Med Singapore. 2009; 38:937-42. [PubMed: 19956814]

59. Zhang Z, Cherryholmes G, Mao A, Marek C, Longmate J, Kalos M, Amand RP, Shively JE. High plasma levels of MCP-1 and eotaxin provide evidence for an immunological basis of fibromyalgia. Experimental biology and medicine. 2008; 233:1171-80. [PubMed: 18535166] 


\section{Perspective}

In US adults, pain occurs more frequently in lower income groups, although the relationship is not attributable to their experience of greater allostatic load. While allostatic load contributes to population variation in pain, other etiologic mechanisms contributing to pain are needed to account for income-disparities in pain. 


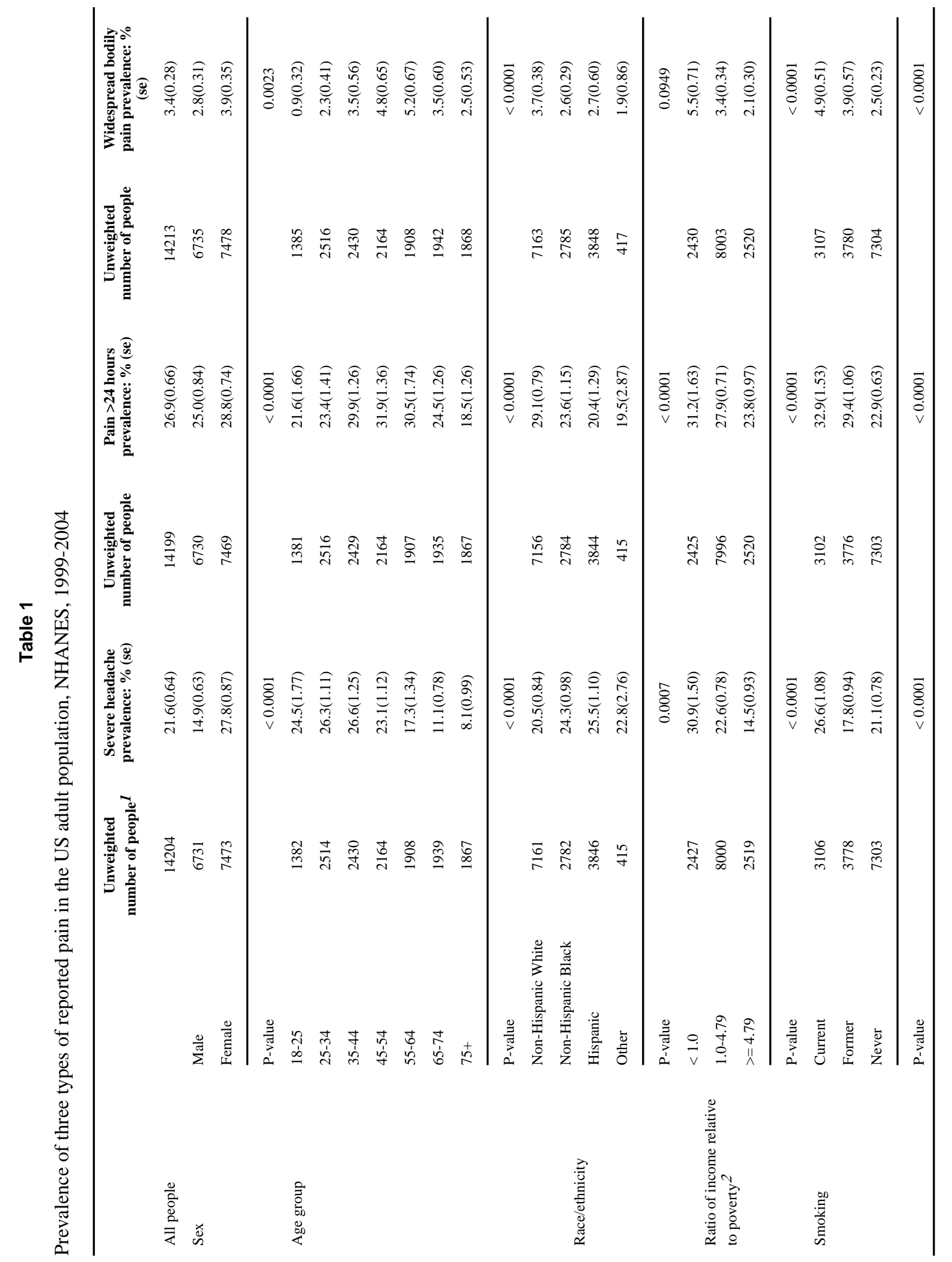




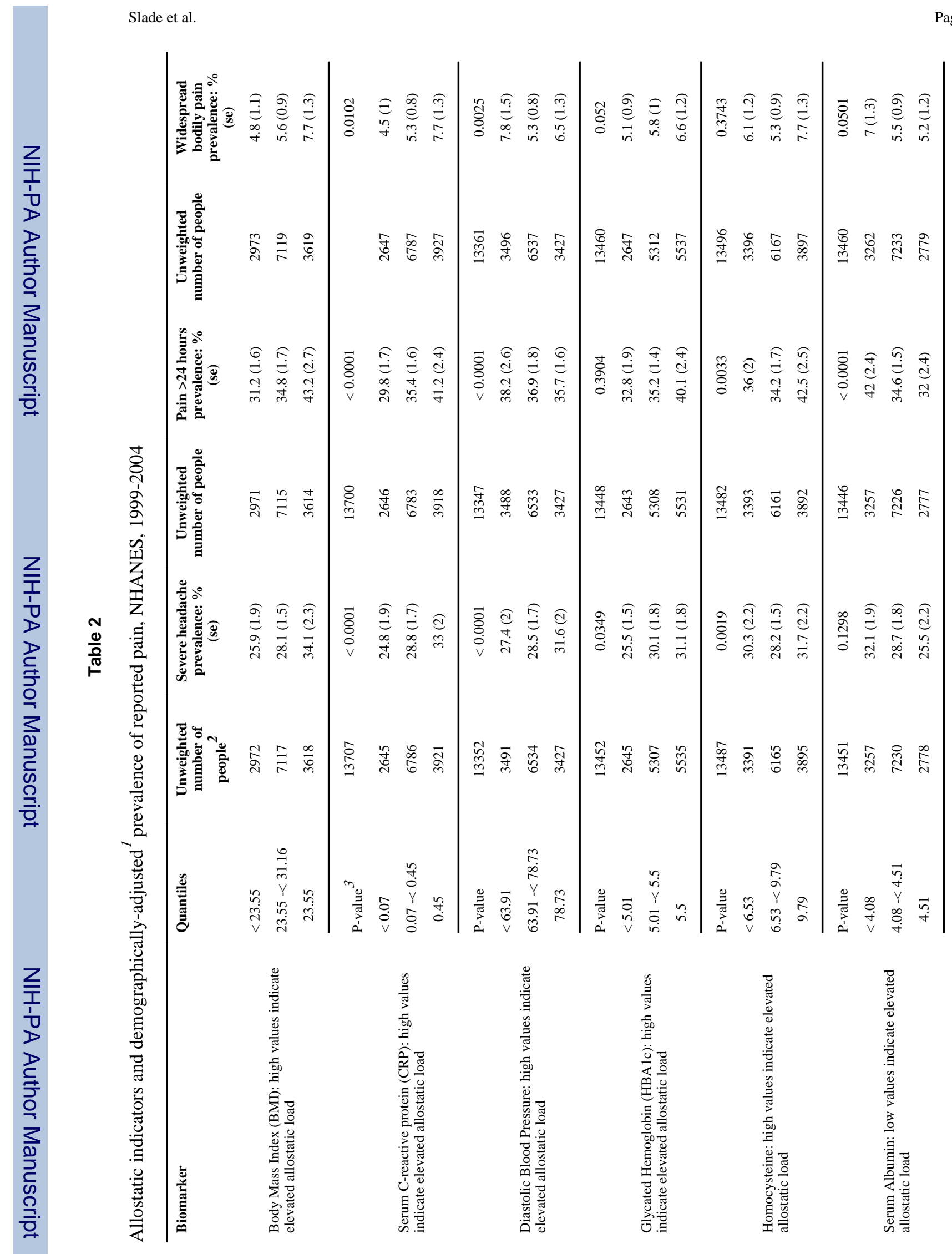

J Pain. Author manuscript; available in PMC 2013 July 01. 


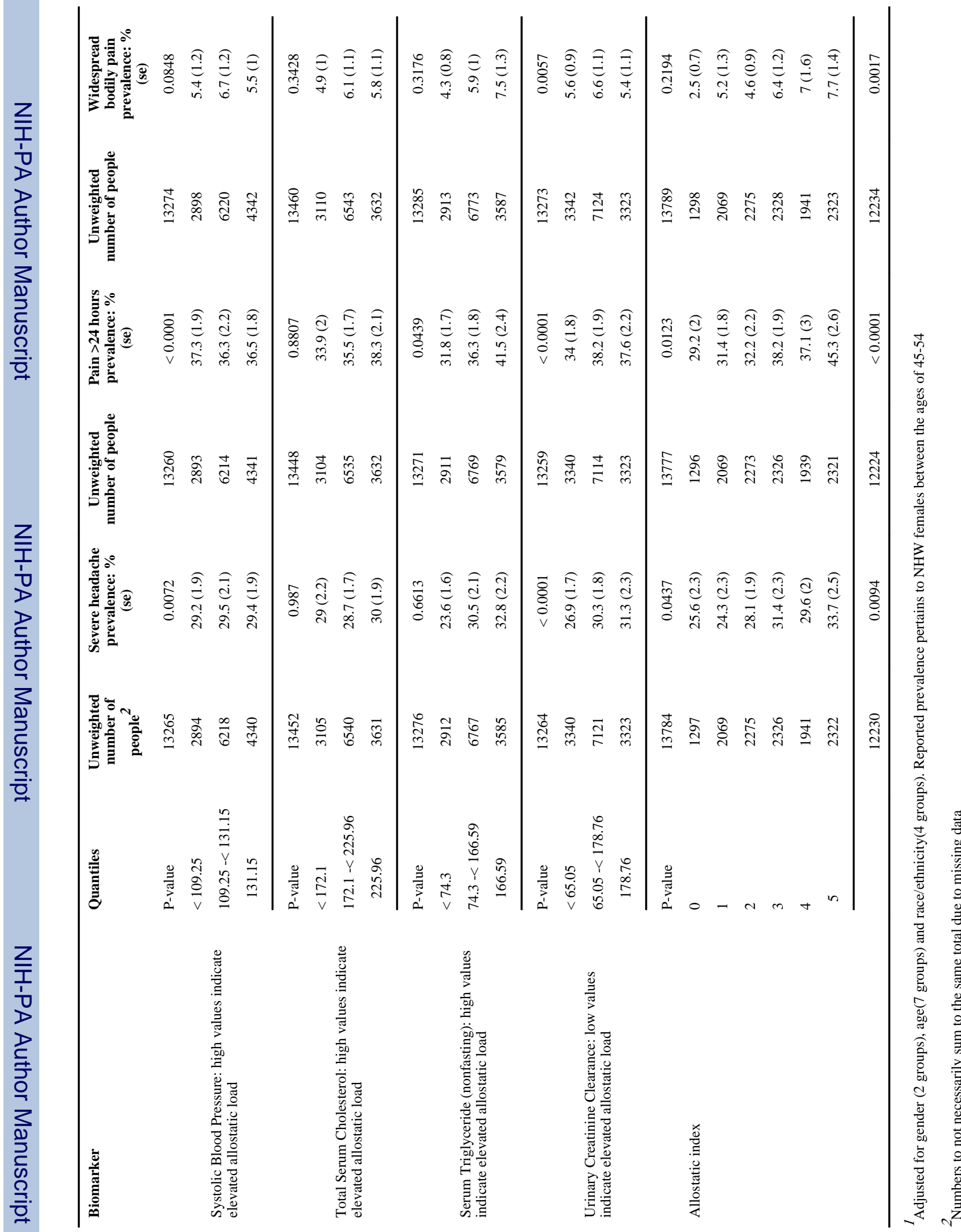


Table 3

Adjusted prevalence ratios for three types of reported pain, NHANES 1999-2004

\begin{tabular}{|c|c|c|c|c|}
\hline & & \multicolumn{3}{|c|}{ Prevalence Ratio $\left(95 \%\right.$ CI) for Severe Headache ${ }^{d}$} \\
\hline & & Model $1^{a}$ & Model $2^{b}$ & Model $3^{c}$ \\
\hline \multirow[t]{2}{*}{ Poverty:Income ratio (reference group = highest) } & Low & $2.05(1.68,2.50)$ & $1.96(1.60,2.40)$ & $1.91(1.57,2.31)$ \\
\hline & Mid & $1.60(1.35,1.89)$ & $1.57(1.32,1.86)$ & $1.53(1.29,1.82)$ \\
\hline \multirow[t]{3}{*}{ Race (reference group =Non-Hispanic White) } & Non-Hispanic Black & $1.00(0.88,1.13)$ & $1.01(0.89,1.14)$ & $0.99(0.87,1.12)$ \\
\hline & Hispanic & $0.99(0.84,1.16)$ & $1.01(0.85,1.19)$ & $1.00(0.85,1.18)$ \\
\hline & Other & $0.93(0.70,1.24)$ & $0.95(0.71,1.28)$ & $0.95(0.70,1.28)$ \\
\hline \multirow{2}{*}{ Smoking (reference group = Never smoked) } & Current & & $1.17(1.02,1.33)$ & $1.16(1.01,1.32)$ \\
\hline & Former & & $1.04(0.94,1.15)$ & $1.03(0.93,1.15)$ \\
\hline \multirow[t]{7}{*}{ Allostasis index (reference group $=0$ ) } & 1 & & & $0.94(0.77,1.15)$ \\
\hline & 2 & & & $1.04(0.88,1.21)$ \\
\hline & 3 & & & $1.20(1.00,1.43)$ \\
\hline & 4 & & & $1.12(0.92,1.37)$ \\
\hline & $>=5$ & & & $1.23(1.02,1.47)$ \\
\hline & & \multicolumn{3}{|c|}{ Prevalence Ratio (95\% CI) for Pain > 24 hours $^{d}$} \\
\hline & & Model $1^{a}$ & Model $2^{b}$ & Model $3^{c}$ \\
\hline \multirow[t]{2}{*}{ Poverty:Income ratio (reference group = highest) } & Low & $1.65(1.49,1.83)$ & $1.57(1.41,1.75)$ & $1.50(1.34,1.68)$ \\
\hline & Mid & $1.31(1.19,1.45)$ & $1.27(1.15,1.40)$ & $1.24(1.12,1.37)$ \\
\hline \multirow{3}{*}{ Race (reference group =Non-Hispanic White) } & Non-Hispanic Black & $0.74(0.65,0.84)$ & $0.75(0.66,0.85)$ & $0.74(0.65,0.84)$ \\
\hline & Hispanic & $0.65(0.55,0.76)$ & $0.66(0.56,0.79)$ & $0.66(0.56,0.79)$ \\
\hline & Other & $0.71(0.50,0.99)$ & $0.73(0.52,1.03)$ & $0.73(0.51,1.03)$ \\
\hline \multirow[t]{2}{*}{ Smoking (reference group = Never smoked) } & Current & & $1.28(1.15,1.43)$ & $1.27(1.14,1.42)$ \\
\hline & Former & & $1.20(1.11,1.30)$ & $1.20(1.11,1.30)$ \\
\hline \multirow[t]{7}{*}{ Allostasis index (reference group=0) } & 1 & & & $1.06(0.92,1.23)$ \\
\hline & 2 & & & $1.06(0.88,1.27)$ \\
\hline & 3 & & & $1.25(1.10,1.42)$ \\
\hline & 4 & & & $1.24(1.02,1.50)$ \\
\hline & $>=5$ & & & $1.45(1.22,1.73)$ \\
\hline & & \multicolumn{3}{|c|}{ Prevalence Ratio (95\% CI) for Widespread Bodily Pain ${ }^{d}$} \\
\hline & & Model $1^{a}$ & Model $2^{b}$ & Model $3^{c}$ \\
\hline \multirow[t]{2}{*}{ Poverty:Income ratio (reference group = highest) } & Low & $3.67(2.56,5.27)$ & $3.15(2.27,4.38)$ & $2.97(2.16,4.10)$ \\
\hline & Mid & $1.93(1.38,2.72)$ & $1.78(1.28,2.47)$ & $1.71(1.23,2.39)$ \\
\hline \multirow[t]{3}{*}{ Race (reference group =Non-Hispanic White) } & Non-Hispanic Black & $0.50(0.35,0.72)$ & $0.52(0.36,0.74)$ & $0.50(0.36,0.71)$ \\
\hline & Hispanic & $0.61(0.37,1.03)$ & $0.66(0.39,1.10)$ & $0.66(0.40,1.09)$ \\
\hline & Other & $0.26(0.06,1.10)$ & $0.28(0.07,1.21)$ & $0.27(0.06,1.17)$ \\
\hline Smoking (reference group = Never smoked) & Current & & $1.78(1.29,2.47)$ & $1.75(1.27,2.42)$ \\
\hline
\end{tabular}

J Pain. Author manuscript; available in PMC 2013 July 01. 


\begin{tabular}{|c|c|c|c|c|}
\hline \multirow{8}{*}{ Allostasis index (reference group $=0$ ) } & \multirow[b]{3}{*}{ Former } & \multicolumn{3}{|c|}{ Prevalence Ratio $\left(95 \%\right.$ CI) for Widespread Bodily Pain ${ }^{d}$} \\
\hline & & \multirow[t]{2}{*}{ Model $1^{a}$} & Model $2^{b}$ & Model $3^{c}$ \\
\hline & & & \multirow[t]{6}{*}{$1.25(0.84,1.86)$} & $1.27(0.85,1.89)$ \\
\hline & 1 & & & $2.44(1.26,4.70)$ \\
\hline & 2 & & & $1.93(1.01,3.68)$ \\
\hline & 3 & & & $2.49(1.35,4.59)$ \\
\hline & 4 & & & $2.99(1.46,6.12)$ \\
\hline & $>=5$ & & & $2.83(1.49,5.35)$ \\
\hline \multicolumn{5}{|c|}{ 'Covariates include age(7 groups), gender(2 groups), race/ethn(4), income-poverty-ratio (3). } \\
\hline \multicolumn{5}{|c|}{${ }^{b}$ Same covariates as Model 1 plus smoking (3 groups). } \\
\hline \multicolumn{5}{|c|}{ Same covariates as Model 2 plus allostatic load index (6 groups). } \\
\hline${ }^{d}$ Severe Headache: $\mathrm{n}=11202 ;$ Pain $>$ & $1197 ; \mathrm{V}$ & ly Pain: $n$ & & \\
\hline
\end{tabular}

